\title{
FURUTA INEQUALITY OF INDEFINITE TYPE
}

\author{
TAKASHI SANO
}

Abstract. In this article, we study matrix inequalities on an (indefinite) inner product space, including a generalization of Furuta inequality: let $A, B$ be $J$-selfadjoint matrices with nonnegative eigenvalues and $I \geqq{ }^{J} A \geqq{ }^{J} B$. Then for each $r \geqq 0$,

$$
\left(A^{\frac{r}{2}} A^{p} A^{\frac{r}{2}}\right)^{\frac{1}{q}} \geqq J\left(A^{\frac{r}{2}} B^{p} A^{\frac{r}{2}}\right)^{\frac{1}{q}}
$$

holds for $p \geqq 0, q \geqq 1$ with $(1+r) q \geqq p+r$.

Mathematics subject classification (2000): 47B50, 47A63.

Key words and phrases: matrix inequality, (indefinite) inner product space, Furuta inequality.

\section{REFERENCES}

[1] T. ANDo, Linear Operators on Krein Spaces, Hokkaido Univ., Sapporo, Japan, 1979.

[2] T. ANDO, Löwner inequality of indefinite type, Linear Algebra Appl., 385, (2004), 73-80.

[3] T. YA. AZIZOV, I. S. IOKHVIDOv, Linear Operators in Spaces with an Indefinite Metric, Nauka, Moscow 1986 English translation: Wiley, New York, 1989.

[4] R. BHATIA, Matrix Analysis, Springer-Verlag, 1997.

[5] T. FURUTA, An elementary proof of an order preserving inequality, Proc. Japan Acad. Ser A. Math. Sci., 65, (1989), 126.

[6] T. FURUTA, Extensions of the Furuta inequality and Ando-Hiai log majorization, Linear Algebra Appl., 219, (1995), 139-155. 